\title{
Raised troponin I in Chinese patients with acute pulmonary embolism suggests complicated clinical course and may predict intensive care unit admission
}

\author{
Pui-Lok Ng${ }^{1}$, MBBS, Chi-Hang Kwok ${ }^{2}$, MBBS, MrCP, Sik-Hon $\underline{T s u i}^{1}$, FRCPE, FRCSE, Hon-Kuan Tong ${ }^{1}$, FCSHK, FHKCEM
}

INTRODUCTION Acute pulmonary embolism (PE) is an uncommon but potentially fatal disease. Acute right ventricular failure, which can be demonstrated by echocardiography, is known to be an adverse prognostic factor in patients with acute PE. However, this diagnostic test is not always available in emergency departments and it is also an operatordependent investigation. This study aimed to investigate whether cardiac troponin I (cTnI) levels could predict clinical outcomes in Chinese patients with PE.

METHODS This was a retrospective cohort study performed in a tertiary regional hospital in Hong Kong. For this study, 100 patients who were diagnosed with acute PE between January 1, 2002 and December 31, 2009 were recruited. Information, including demographic data, presenting symptoms and vital signs at presentation, predisposing factors for PE, results of diagnostic procedures and clinical outcomes, was collected from the medical records of these patients.

RESULTS $71 \%$ of recruited patients had elevated cTnl levels. High cTnl levels were associated with haemodynamic instability (odds ratio [OR] 5.30, 95\% confidence interval [CI] 1.32-27.71; $\mathrm{p}=0.019$ ) and complicated clinical course (OR 6.34, 95\% Cl 1.76-22.9; $\mathrm{p}=0.002$ ).

CONCLUSION Elevated cTnl level was associated with a complicated clinical course in patients with acute PE. We suggest that measurements of cTnl levels be used for the early risk stratification of patients with PE in the emergency departments of hospitals.

Keywords: cTnI, Chinese, PE, pulmonary embolism, troponin I

Singapore Med J 2013; 54(2): 86-89

\section{INTRODUCTION}

Venous thromboembolism (VTE) is a spectrum of diseases that includes deep vein thrombosis (DVT) and pulmonary embolism (PE). PE is a potentially fatal complication of DVT. Despite recent advances in the diagnosis and treatment of $\mathrm{PE}$, the risk stratification of cardiopulmonary mortality in patients with PE remains difficult. Haemodynamic instability is associated with in-hospital mortality, which can be as high as $50 \%$ in patients with PE. ${ }^{(1)}$ Among patients having acute PE with normal blood pressure at presentation, acute right ventricular (RV) failure is a poor prognostic factor. ${ }^{(1)} \mathrm{RV}$ failure can be demonstrated by echocardiography, which shows RV enlargement and hypokinesis. ${ }^{(2)}$ However, this test is not always available in the emergency departments of hospitals, and it also depends heavily on the experience and capability of the operators. Plasma cardiac troponin I (cTnl) is a highly sensitive and specific biochemical marker for myocardial injury. ${ }^{(3)}$ Elevated cTnl levels are observed in patients with acute PE, especially in those with massive PE. In addition, Tnl level has prognostic implications, as it reflects myocardial injury and RV dysfunction. ${ }^{(4-6)}$

VTE is common in Caucasians, with an annual estimated incidence of 117 cases per 100,000 persons in the United States. ${ }^{(7)}$ It is less common among Asians, including persons of Chinese ancestry. A local study has found that the annual incidence of VTE among Hong Kong Chinese patients was 16.6 per 100,000 population. ${ }^{(8)}$ One possible explanation for the lower incidence of VTE seen among Chinese patients may be related to the prevalence of activated protein $\mathrm{C}$ resistance, which is extremely low in Chinese populations. ${ }^{(9)}$ However, evidence of the prognostic value of cTnl in Chinese patients with PE is scarce. Therefore, this retrospective study was conducted to assess whether cTnl levels could predict clinical outcomes and complications in Chinese patients with PE.

\section{METHODS}

This was a retrospective study carried out between January 1, 2002 and December 31, 2009 at the Princess Margaret Hospital, Hong Kong, China, a tertiary regional hospital. The research complied with the Declaration of Helsinki and was approved by the local clinical research ethics committee.

The medical records of patients older than 18 years of age who were admitted for the management of acute PE were reviewed. Patients who satisfied any of the following three criteria were excluded from the study: (a) recent acute coronary syndrome within six months; (b) end-stage renal failure (creatinine clearance $<15 \mathrm{~mL} / \mathrm{min}$ ); and (c) no cTnl investigations performed.

${ }^{1}$ Department of Accident and Emergency, Queen Mary Hospital, ${ }^{2}$ Department of Medicine and Geriatrics, Princess Margaret Hospital, Hong Kong, China Correspondence: Dr Pui-Lok Ng, Resident, Department of Accident and Emergency, Queen Mary Hospital, 102 Pok Fu Lam Road, Pokfulam, Hong Kong Island, Hong Kong, China. lucypl@yahoo.com 
Table I. Baseline characteristics of the study population.

\begin{tabular}{|c|c|c|c|c|}
\hline \multirow[t]{2}{*}{ Variable } & \multicolumn{3}{|c|}{ No. of patients (\%) } & \multirow[t]{2}{*}{ p-value } \\
\hline & $\mathrm{cTnl} \leq 0.04 \mathrm{ng} / \mathrm{ml}(\mathrm{n}=29)$ & $\mathrm{cTnl}>0.04 \mathrm{ng} / \mathrm{ml}(\mathrm{n}=71)$ & Total $(n=100)$ & \\
\hline Male & $12(41.4)$ & $36(50.7)$ & $48(48.0)$ & NS \\
\hline Median age (IQR) [yrs] & $61.0(45.0-80.5)$ & $75.0(49.0-81.0)$ & $68.5(48.0-81.0)$ & NS \\
\hline \multicolumn{5}{|l|}{ Risk factors of PE } \\
\hline Immobilisation & $15(51.7)$ & $25(35.2)$ & $40(40.0)$ & NS \\
\hline Malignancy & $10(34.5)$ & $13(18.3)$ & $23(23.0)$ & NS \\
\hline Recent surgery & $9(31.0)$ & $14(19.7)$ & $23(23.0)$ & NS \\
\hline Thrombophilia & $1(11.1)^{*}$ & $5(15.2)^{*}$ & $6(14.3)^{*}$ & NS \\
\hline Concurrent DVT & $13(59.1)^{\dagger}$ & $27(52.9)^{\dagger}$ & $40(54.8)^{\dagger}$ & NS \\
\hline \multicolumn{5}{|l|}{ History } \\
\hline PE & $1(3.4)$ & $1(1.4)$ & $2(2.0)$ & NS \\
\hline DVT & $3(10.3)$ & $4(5.6)$ & $7(7.0)$ & NS \\
\hline Hypertension & $9(31.0)$ & $28(39.4)$ & $37(37)$ & NS \\
\hline Diabetes mellitus & $3(10.3)$ & $13(18.3)$ & $16(16.0)$ & NS \\
\hline COPD & $2(6.9)$ & $6(8.5)$ & $8(8.0)$ & NS \\
\hline
\end{tabular}

${ }^{*}$ A total of 42 patients were screened for thrombophilia, of whom 9 patients had normal cTnl levels and 33 patients had elevated cTnl.

${ }^{\dagger}$ A total of 73 patients underwent lower limb Doppler ultrasonography screening, of whom 22 patients had normal cTnl levels and 51 patients had elevated cTnl.

cTnI: cardiac troponin I; IQR: interquartile range; PE: pulmonary embolism; DVT: deep vein thrombosis; NS: not significant; COPD: chronic obstructive pulmonary disease

Data extracted from the patients' medical records included demographic information, presenting symptoms, vital signs at presentation, predisposing factors for $\mathrm{PE}$, results of diagnostic procedures, including D-dimers, first cTnI level, arterial blood gas analysis, electrocardiography (ECG), transthoracic echocardiography, spiral computed tomography pulmonary angiography (CT-PA), ventilation-perfusion (V/P) scan and lower extremity duplex ultrasonography. Patients were assigned to two groups based on their observed cTnl levels (cTnl $\leq 0.04 \mathrm{ng} / \mathrm{mL}$ or cTnl $>0.04 \mathrm{ng} / \mathrm{mL}$ ). Outcome parameters, including in-hospital mortality, length of hospital stay and complicated clinical course, were examined. Complicated clinical course was defined as at least one of the following: (a) use of thrombolytic therapy; (b) need for mechanical ventilation; or (c) need for inotropic support. ${ }^{(10)}$

$\mathrm{S}_{1} \mathrm{Q}_{3} \mathrm{~T}_{3}$ and right bundle branch block were taken as ECG features of RV strain. Echocardiographic findings of RV dysfunction included paradoxical interventricular septal motion, RV dilation (diastolic diameter $>15 \mathrm{~mm} / \mathrm{m}^{2}$ ) and low RV systolic function (systolic excursion of the tricuspid annulus $<15 \mathrm{~mm}$ ). ${ }^{(11)}$ cTnl was determined by a specific immunoassay (Access AccuTnl Assay, Beckman Coulter, Brea, CA USA). cTnl level > 0.04 ng/mL (upper limit of the 99th percentile in the normal population) was considered to be elevated in accordance with the local laboratory normal reference ranges. The D-dimer levels were determined by an enzyme-linked immunosorbent assay using an analyser (mini VIDAS analyser, bioMérieux, Marcy l'Etoile, France). The diagnosis of PE was established based on the evidence of a filling defect on contrast-enhanced spiral CT-PA or high-probability lung scintigraphy (V/Q scan).

The five major clinical outcomes measured in our study were: (a) death; (b) haemodynamic instability (defined as systolic blood pressure $[\mathrm{BP}]<90 \mathrm{mmHg}$, or a BP drop $>40 \mathrm{mmHg}$ for $\geq 15$ minutes, with signs of organ hypoperfusion not due to hypovolaemia or sepsis); (c) need of thrombolytic therapy; (d) admission to high-dependency units (intensive care units [ICUs] or coronary care units); and (e) length of hospital stay. ${ }^{(12)}$ Recurrent PE was defined as new or worsened signs and symptoms, with repeat CT-PA demonstrating a new filling defect of a different pulmonary artery.

The Statistical Package for the Social Sciences for Windows version 16.0 (SPSS Inc, Chicago, IL, USA) was used for statistical analysis. The data were expressed as mean \pm standard deviation (SD), median with interquartile range (IQR) or proportion with 95\% confidence intervals (Cls). The difference between the demographic and clinical parameters of the two patient groups was examined using Fisher's exact or chi-square tests for categorical variables. The Mann-Whitney $U$ test and Student's $t$-test were used to determine the statistical significance of continuous variables. Multivariate logistic regression analysis was used to determine the association between elevated cTnl levels and mortality, after adjusting for confounders such as age, gender, malignancy, chronic lung diseases and history of arterial cardiovascular diseases. The automatic stepwise forward (likelihood ratio test) modality was used in the regression model. A p-value $<0.05$ was considered to be statistically significant.

\section{RESULTS}

During the study period, 110 Chinese patients were admitted for acute PE. Of these, 10 (9.1\%) patients were excluded from the study due to unavailability of $\mathrm{cTnl}$ investigations. Of the 100 $(90.9 \%)$ patients that were finally recruited in the study, 48 $(48.0 \%)$ were men. The median age of the patients at diagnosis was 68.5 (IQR 48.0-81.0) years. The diagnosis of PE was confirmed using contrast CT imaging in $98(98.0 \%)$ patients and by high-probability lung scintigraphy in 2 (2.0\%) patients. Doppler ultrasonography of the lower limbs was performed in 73 (73.0\%) patients, and among these, 40 (54.8\%) patients were diagnosed to have concurrent DVT.

The baseline characteristics of patients are summarised in Table I. There was no significant difference in the baseline 
Table II. Clinical outcomes of patients.

\begin{tabular}{|c|c|c|c|c|c|}
\hline \multirow[t]{2}{*}{ Variable } & \multicolumn{3}{|c|}{ No. of patients (\%) } & \multirow[t]{2}{*}{ OR $(95 \% \mathrm{Cl})$} & \multirow[t]{2}{*}{ p-value } \\
\hline & $c T n l \leq 0.04(n=29)$ & $c T n l>0.04(n=71)$ & Total $(n=100)$ & & \\
\hline RV strain pattern on ECG & $3(10.3)$ & $20(28.2)$ & $23(23.0)$ & & 0.055 \\
\hline Creatine kinase* (IU/L) & $54.5(44.3-113.8)$ & $86.0(60.0-134.0)$ & $85.0(53.0-124.5)$ & & 0.035 \\
\hline Complicated clinical course & $3(10.3)$ & $30(42.3)$ & $33(33.0)$ & $6.3(1.8-22.9)$ & 0.005 \\
\hline Thrombolytic therapy & $0(0.0)$ & $12(16.9)$ & $12(12.0)$ & $1.5(1.3-1.7)$ & 0.017 \\
\hline ICU admission & $3(10.3)$ & $22(31.0)$ & $25(25.0)$ & $3.9(1.1-14.2)$ & 0.031 \\
\hline Death & $2(6.9)$ & $13(18.3)$ & $15(15.0)$ & & NS \\
\hline Shock & $3(10.3)$ & $20(28.2)$ & $23(23.0)$ & $3.4(0.9-12.5)$ & $0.055^{+}$ \\
\hline Length of hospital stay (days) & $11(9.0-15.0)$ & $15(10.0-23.3)$ & $13(9.5-20.0)$ & & 0.021 \\
\hline
\end{tabular}

${ }^{*}$ Data is presented as median (interquartile range). ${ }^{\dagger} p>0.05$ was not significant.

RV: right ventricular; ECG: electrocardiography; ICU: intensive care unit; CI: confidence interval; OR: odds ratio; NS: not significant

Table III. Risk factors of haemodynamic instability.

\begin{tabular}{lll}
\hline Risk factor & OR $(\mathbf{9 5 \%} \mathbf{C l})$ & p-value \\
\hline $\begin{array}{l}\text { Univariate analysis } \\
\quad \text { Elevated cTnl }\end{array}$ & $3.4(0.9-12.5)$ & 0.066 \\
$\begin{array}{l}\text { Multivariate analysis* } \\
\text { Elevated cTnl }\end{array}$ & \\
$\quad$ Malignancy & $5.3(1.3-27.7)$ & 0.019 \\
\hline
\end{tabular}

* Logistic regression analysis; ORs were adjusted for potential confounders such as age, gender, chronic lung diseases and history of arterial cardiovascular diseases.

$\mathrm{Cl}$ : confidence interval; cTnl: cardiac troponin I; OR: odds ratio

Table IV. Risk factors of in-hospital mortality.

\begin{tabular}{llc}
\hline Risk factor & OR (95\% Cl) & p-value \\
\hline $\begin{array}{lll}\text { Univariate analysis } \\
\text { Elevated cTnl }\end{array}$ & $3.4(0.9-12.5)$ & 0.219 \\
$\begin{array}{l}\text { Haemodynamic instability } \\
\text { at presentation }\end{array}$ & $6.8(1.2-37.8)$ & 0.042 \\
$\begin{array}{l}\text { Malignancy } \\
\text { Multivariate analysis* }\end{array}$ & $5.3(1.7-17.0)$ & 0.006 \\
Elevated cTnl & $5.1(0.9-28.4)$ & 0.066 \\
\hline
\end{tabular}

*Logistic regression analysis; ORs were adjusted for potential confounders such as age, gender, chronic lung diseases, history of arterial cardiovascular diseases and active malignancy.

$\mathrm{Cl}$ : confidence interval; cTnl: cardiac troponin I; OR: odds ratio

characteristics of patients with cTnl $\leq 0.04 \mathrm{ng} / \mathrm{mL}$ and those with $\mathrm{cTnl}>0.04 \mathrm{ng} / \mathrm{mL}$. $71(71.0 \%)$ patients were found to have elevated cTnl levels at presentation. 57 patients were diagnosed with PE at presentation - 6 (10.9\%) patients were diagnosed with massive PE (haemodynamic instability), 18 (32.7\%) patients with submassive PE (normal vital signs but RV dysfunction) and 31 (56.4\%) patients with minor PE (normal vital signs and RV function), according to the Goldhaber classification. ${ }^{(13)}$ The remaining $43(43.0 \%)$ patients' $\mathrm{PE}$ status at presentation could not be determined, as echocardiography was not performed. The mortality rate and proportion of patients who had elevated cTnl levels were much higher among patients with massive PE (mortality 50.0\%; elevated cTnl 100\%) than among those with minor PE (mortality 16.1\%; elevated cTnl $67.7 \%$ ). Patients with elevated cTnl levels presented to the emergency departments sooner than those with normal cTnl (median 24 hours vs. 48 hours, $\mathrm{p}=0.01)$.
A total of $30(42.3 \%)$ patients with elevated cTnl levels had a complicated clinical course, whereas only 3 (10.3\%) patients with normal cTnl had such complications. The relative risk for complicated clinical course in patients with elevated cTnl was $6.3(95 \% \mathrm{Cl} 1.8-22.9 ; \mathrm{p}=0.005)$. The sensitivity and specificity of the cTnl test for complicated clinical course were $90.9 \%$ and $38.8 \%$, respectively. The negative predictive and positive predictive values of high cTnl level for complicated clinical course were $89.7 \%$ and $57.7 \%$, respectively. An elevated cTnl level was found to predict poor clinical outcome in patients who were haemodynamically stable at presentation (odds ratio [OR] 7.3, 95\% Cl 1.8-30.6; $p=0.006)$. It was also associated with a higher creatine kinase level, ECG findings of RV strain patterns, need for thrombolytic therapy, ICU admission and longer length of hospital stay (Table II).

On univariate analysis, elevated cTnl levels were associated with haemodynamic instability (Table III). Subsequent multivariate logistic regression analysis showed that the risk of developing haemodynamic instability was independently associated with elevated cTnl levels, independent of age and malignancy. The OR of elevated cTnl was 5.3 (95\% Cl 1.3-27.7; $p=0.019) .15$ (15.0\%) patients died during the hospitalisation period. Haemodynamic instability at presentation and malignancy were associated with in-hospital mortality on univariate analysis (Table IV). However, cTnl levels failed to demonstrate a statistically significant association with in-hospital mortality on univariate analysis. After adjusting for potential confounders, including haemodynamic instability at presentation and malignancy, it was found that elevated cTnl might be weakly associated with higher in-hospital mortality (OR 5.1, 95\% Cl 0.9-28.4; $\mathrm{p}=0.066$ ).

\section{DISCUSSION}

According to Goldhaber, hypotension and RV overload, which can be assessed by echocardiography, can be used to stratify patients with acute PE for the risk of death. ${ }^{(13)}$ However, 24-hour echocardiography by experienced personnel is a prerequisite for accurate diagnosis, and disagreement exists regarding the criteria for RV dysfunction. Other studies have shown that cTnl is a highly sensitive marker for RV dysfunction and suggested that elevated cTnl levels are associated with mortality and adverse clinical 
outcome. ${ }^{(14-16)}$ The findings of our study are consistent with these earlier reports.

In the present study, cTnl was found to predict complicated clinical course and haemodynamic instability in Chinese patients with acute PE. Its prognostic value was also observed in patients who were haemodynamically stable at presentation. However, this study failed to demonstrate a statistically significant relationship between elevated cTnl levels and in-hospital mortality $(p=0.066)$. In our study, the time lapse between the onset of symptoms of first acute PE episode and presentation at the hospital was markedly shorter in patients with elevated cTnl (24 hours vs. 48 hours, $p=0.01$ ). Punukollu et al, who found that no patient with a duration of symptoms $>72$ hours would have raised cTnl in spite of the presence of RV dysfunction, concluded that the dynamics of cTnl release are different between patients with duration of symptoms $>72$ hours and those with a shorter duration of symptoms. ${ }^{(17)}$ Therefore, it is possible that a patient with acute PE who presents late to a hospital may have a normal cTnl level on admission even though RV dysfunction is present. In our study, the duration of symptoms was longer than 72 hours for 17 (17.0\%) patients who presented with PE. Clinicians should bear in mind that cTnl might not predict the prognosis for these patients.

Our study also revealed that the negative predictive value of cTnl for complicated clinical course was high (89.7\%). This finding is consistent with those by Konstantinides et $\mathrm{al}^{(6)}$ and Kucher et $\mathrm{al}^{\left({ }^{(4)}\right.}$ who reported slightly higher negative predictive values of $93 \%$ and $95 \%$, respectively. Recent studies have shown that repeat cTnl measurement has additional prognostic value. According to Pruszczyk et al ${ }^{(18)}$ and Müller-Bardorff et al, ${ }^{(19)}$ patients with acute PE may have normal cTnl levels at presentation, but show elevated cTnl later at 6 and 12 hours. We suggest that cTnl measurement be performed for all patients presenting with acute PE whose duration of symptoms is less than 72 hours. cTnl measurements should be repeated at 6 and 12 hours for such patients, if the initial cTnl readings are normal. In our study, elevated cTnl levels were associated with complicated clinical course in patients with $\mathrm{PE}$, and therefore, prompt thrombolytic therapy and intensive care are warranted for these patients.

Our study, however, was not without limitations. As this was a retrospective study, it was thus subject to selection bias and recall bias. Investigations of the association between raised cTnl and RV dysfunction were limited, as only $53 \%$ of our patients had undergone echocardiography. Our modest sample size also limited the power of the study with regard to in-hospital mortality. Furthermore, it was difficult to exclude concomitant acute coronary syndromes in patients who had raised cTnl but no acute ischaemic changes on ECG. Finally, patients with very massive PE who died in the emergency department on or before arrival, for whom autopsy was not performed, were excluded from the study.
In conclusion, we found that elevated cTnl levels were predictive for a subgroup of patients with acute PE who were prone to develop haemodynamic instability and complications in the clinical course during hospitalisation. We propose that cTnl be used as a marker for the early risk stratification of patients presenting with acute PE to the emergency departments of hospitals.

\section{REFERENCES}

1. Goldhaber SZ, Visani L, De Rosa M. Acute pulmonary embolism: clinical outcomes in the International Cooperative Pulmonary Embolism Registry (ICOPER). Lancet 1999; 353:1386-9.

2. Kasper W, Konstantinides S, Geibel A, et al. Prognostic significance of right ventricular afterload stress detected by echocardiography in patients with clinically suspected pulmonary embolism. Heart 1997; 77:346-9.

3. Alpert JS, Thygesen K, Antman E, Bassand JP. Myocardial infarction redefined--a consensus document of The Joint European Society of Cardiology/American College of Cardiology Committee for the redefinition of myocardial infarction. J Am Coll Cardiol 2000; 36:959-69.

4. Kucher N, Wallmann D, Carone A, et al. Incremental prognostic value of troponin I and echocardiography in patients with acute pulmonary embolism. Eur Heart J 2003; 24:1651-6.

5. Mehta NJ, Jani K, Khan IA. Clinical usefulness and prognostic value of elevated cardiac troponin I levels in acute pulmonary embolism. Am Heart J 2003; 145:821-5.

6. Konstantinides S, Geibel A, Olschewski M, et al. Importance of cardiac troponins $\mathrm{I}$ and $\mathrm{T}$ in risk stratification of patients with acute pulmonary embolism. Circulation 2002; 106:1263-8.

7. Silverstein MD, Heit JA, Mohr DN, et al. Trends in the incidence of deep vein thrombosis and pulmonary embolism: a 25-year population-based study. Arch Intern Med 1998; 158:585-93.

8. Liu HS, Kho BC, Chan JC, et al. Venous thromboembolism in the Chinese population--experience in a regional hospital in Hong Kong. Hong Kong Med J 2002; 8:400-5.

9. Chan LC, Bourke C, Lam CK, et al. Lack of activated protein C resistance in healthy Hong Kong Chinese blood donors-correlation with absence of Arg506-GIn mutation of factor $V$ gene. Thromb Haemost 1996; 75:522-3.

10. Aksay E, Yanturali S, Kiyan S. Can elevated troponin I levels predict complicated clinical course and inhospital mortality in patients with acute pulmonary embolism? Am J Emerg Med 2007; 25:138-43.

11. Kasper W, Konstantinides S, Geibel A, et al. Management strategies and determinants of outcome in acute major pulmonary embolism: results of a multicenter registry. J Am Coll Cardiol 1997; 30:1165-71.

12. Torbicki A, Perrier A, Konstantinides S, et al. Guidelines on the diagnosis and management of acute pulmonary embolism: the Task Force for the Diagnosis and Management of Acute Pulmonary Embolism of the European Society of Cardiology (ESC). Eur Heart J 2008; 29:2276-315.

13. Goldhaber SZ. Pulmonary embolism. Lancet 2004; 363:1295-305.

14. Meyer T, Binder L, Hruska N, Luthe H, Buchwald AB. Cardiac troponin I elevation in acute pulmonary embolism is associated with right ventricular dysfunction. J Am Coll Cardiol 2000; 36:1632-6.

15. Giannitsis E, Müller-Bardorff M, Kurowski V, et al. Independent prognostic value of cardiac troponin $\mathrm{T}$ in patients with confirmed pulmonary embolism. Circulation 2000; 102:211-7.

16. Becattini C, Vedovati MC, Agnelli G. Prognostic value of troponins in acute pulmonary embolism: a meta-analysis. Circulation 2007; 116:427-33

17. Punukollu G, Khan IA, Gowda RM, et al. Cardiac troponin I release in acute pulmonary embolism in relation to the duration of symptoms. Int J Cardiol 2005; 99:207-11.

18. Pruszczyk P, Bochowicz A, Torbicki A, et al. Cardiac troponin T monitoring identifies high-risk group of normotensive patients with acute pulmonary embolism. Chest 2003; 123:1947-52.

19. Müller-Bardorff M, Weidtmann B, Giannitsis E, Kurowski V, Katus HA. Release kinetics of cardiac troponin $\mathrm{T}$ in survivors of confirmed severe pulmonary embolism. Clin Chem 2002; 48:673-5. 